\title{
Algebraic limit cycles in piecewise linear differential systems
}

\author{
Claudio A. Buzzi \\ Departamento de Matemática, Universidade Estadual Paulista, São José do Rio Preto, Brazil \\ buzzi@ibilce.unesp.br \\ Armengol Gasull and Joan Torregrosa \\ Departament de Matemàtiques, Universitat Autònoma de Barcelona, 08193 Bellaterra, Barcelona, Spain \\ gasull@mat.uab.cat,torre@mat.uab.cat \\ Received (to be inserted by publisher)
}

\begin{abstract}
This paper is devoted to study the algebraic limit cycles of planar piecewise linear differential systems. In particular we present examples exhibiting two explicit hyperbolic algebraic limit cycles, as well as some 1-parameter families with a saddle-node bifurcation of algebraic limit cycles. We also show that all degrees for algebraic limit cycles are allowed.
\end{abstract}

Keywords: Non-smooth differential system, piecewise linear differential system, algebraic limit cycle, hyperbolic and double limit cycle, saddle-node bifurcation of limit cycles

\section{Introduction}

The study of algebraic limit cycles of planar polynomial differential equations has a long history. To the best of our knowledge they started to be considered, particularly for quadratic systems, around the late fifties of last Century by the Chinese and Russian schools, see the survey paper [Llibre, 2008] and the references therein. The main motivation of these works was to advance in the knowledge and role of the algebraic solutions of polynomial differential equations and constitutes, somehow, a continuation of the classical works of Darboux and Poincaré in this direction.

For instance, up to now it is known that there are quadratic systems having algebraic limit cycles of degrees 2, 4, 5 or 6, see [Chavarriga et al., 2001; Llibre \& Świrszcz, 2006, 2007] and their references. Notice that in the smooth world, these type of differential equations are the simplest ones that can have this type of solutions.

In this paper we answer some natural questions about the parallel problem of algebraic periodic solutions but in simple piecewise differential systems. We consider two linear systems with a straight line of separation and we study the existence, number and degree of the "algebraic" limit cycles in this setting.

In fact, in non-smooth dynamics the differential equations appearing in the simplest models are linear and of the type described above. These models have attracted the attention of many scientists not only because of its simplicity, but also for the accuracy of the obtained results comparing with the real observations, see more details in [Acary et al., 2011; Brogliato, 2016; Kunze, 2000].

In order to state precisely our results we introduce first some notations and definitions. Consider the piecewise differential system

$$
X^{ \pm}:\left(x^{\prime}, y^{\prime}\right)=\left(f^{ \pm}(x, y), g^{ \pm}(x, y)\right),
$$


defined in $\Sigma^{ \pm}=\left\{(x, y) \in \mathbb{R}^{2}: \pm x \geq 0\right\}$. If an equilibrium point of $X^{ \pm}$is in $\Sigma^{ \pm}$it is said that it is a real equilibrium point for the piecewise differential system. Otherwise it is called a virtual equilibrium point.

Notice that over the line $\Sigma=\left\{(0, y) \in \mathbb{R}^{2}\right\}$ the vector field is bivaluated. We say that a point $\left(0, y_{0}\right) \in \Sigma$ is of crossing type if $f^{+}\left(0, y_{0}\right) f^{-}\left(0, y_{0}\right)>0$. Moreover, if $f^{+}\left(0, y_{0}\right)=0$ or $f^{-}\left(0, y_{0}\right)=0$, and non simultaneously vanishing, then $\left(0, y_{0}\right)$ is called a tangent point. Otherwise, if $\left(0, y_{0}\right)$ is not an equilibrium, it is said of sliding type. All these points define the sliding set. Moreover, the sliding set will be attracting (repelling) if $f^{+}\left(0, y_{0}\right)<0(>0)$ and $f^{-}\left(0, y_{0}\right)>0(<0)$. Periodic orbits that have neither sliding part nor tangent points are called crossing periodic orbits, otherwise they are called sliding periodic orbits. As usual, isolated periodic orbits are called limit cycles. Some examples of sliding limit cycles are showed in Figure 3. For more details on these type of limit cycles and a codimension 1 bifurcation where they can appear see [Guardia et al., 2011; Kuznetsov et al., 2003], or for general properties of piecewise differential systems see [di Bernardo et al., 2008; Filippov, 1988].

Recall that given two limit cycles, $\Gamma_{1}$ and $\Gamma_{2}$, they are nested if $\Gamma_{1} \subset \operatorname{Int}\left(\Gamma_{2}\right)$, or conversely. As usual, for any Jordan curve $\Gamma$, Int $(\Gamma)$ denotes the bounded connected component of $\mathbb{R}^{2} \backslash \Gamma$. Similarly, any number of limit cycles are said to be nested if each two of them are nested.

We say that an isolated periodic orbit $\Gamma$ is an algebraic limit cycle if all its points, except the ones on the sliding set in $\Sigma$, are contained in the level sets of one or two polynomials, that is if $\left(\Gamma \cap \Sigma^{ \pm}\right) \backslash \Sigma \subset\{(x, y) \in$ $\left.\mathbb{R}^{2}: p^{ \pm}(x, y)=0\right\}$ for some irreducible polynomials $p^{-}$and $p^{+}$, that might coincide. If the respective degrees of $p^{-}$and $p^{+}$are $m$ and $n$, we say that the degree of $\Gamma$ is $(m, n)$. For the sake of shortness we write $\Gamma=\left\{p^{-}, p^{+}\right\}$.

As we have already said, in this paper we deal with algebraic limit cycles for piecewise linear differential systems. Before stating our main result we present a simple example of algebraic limit cycle for a piecewise linear differential system with a virtual node for $X^{-}$and a real center for $X^{+}$,

$$
\begin{aligned}
& X^{+}:\left(x^{\prime}, y^{\prime}\right)=(-y, x), \\
& X^{-}:\left(x^{\prime}, y^{\prime}\right)=(6 x-4 y-2,27 x-15 y-12) .
\end{aligned}
$$

With the techniques introduced in this work it can be proved that its unique limit cycle is

$$
\Gamma=\left\{9 x-4 y-5+(3 x-y-2)^{2}, x^{2}+y^{2}-1\right\}
$$

and it is stable, hyperbolic, algebraic and of degree $(2,2)$, see Figure 1.

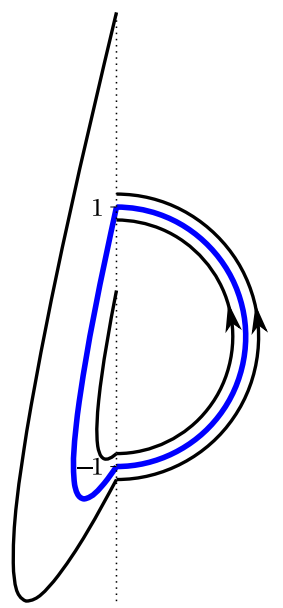

Fig. 1. An algebraic limit cycle of degree $(2,2)$ 
Theorem 1.1. Consider the family of piecewise linear differential systems

$$
X^{ \pm}:\left(x^{\prime}, y^{\prime}\right)=\left(f^{ \pm}(x, y), g^{ \pm}(x, y)\right),
$$

where $f^{ \pm}$and $g^{ \pm}$are polynomials of degree 1 defined in $\Sigma^{ \pm}=\left\{(x, y) \in \mathbb{R}^{2}: \pm x \geq 0\right\}$. The following properties hold:

(i) If some system (2) has an algebraic limit cycle, then each of the corresponding linear smooth systems, $X^{ \pm}$, must be either without equilibrium points or have a unique equilibrium point of one of the three types: saddle, node with different eigenvalues, or center. Moreover, in the first two cases the quotient between the eigenvalues of the Jacobian matrix of the corresponding vector fields at the equilibrium points must be rational numbers.

(ii) All algebraic limit cycles must be of crossing type.

(iii) If a system (2) has an algebraic limit cycle, all the other limit cycles of this system must be algebraic as well. Moreover, all of them are nested.

(iv) There are not algebraic limit cycles $\Gamma=\left\{p^{-}, p^{+}\right\}$such that $p^{-}=p^{+}$.

(v) There are explicit systems (2) with exactly two hyperbolic and algebraic limit cycles.

(vi) There are explicit systems (2) with exactly one explicit double (semistable) algebraic limit cycle.

(vii) For every $(m, n) \in \mathbb{N}^{2}, m \geq 2, n \geq 2$, there is a system (2) with a hyperbolic algebraic limit cycle of degree $(m, n)$.

Notice that with our definition of limit cycle, the periodic orbits such that the bounded region that they delimit is full of periodic orbits and have a neighborhood of its exterior free of periodic orbits are not limit cycles. This situation may happen for piecewise linear differential systems: it suffices to take in one side as $X^{+}$a system with a real center in the interior of $\Sigma^{+}$and in the other side a suitable $X^{-}$. Many times, the ellipse tangent to $\Sigma$ is "almost" an algebraic limit cycle because it is one side isolated in the set of periodic orbits. This orbit is not of crossing type.

Although nowadays it is known that there are systems of type (2) having three (non algebraic) limit cycles, see [Buzzi et al., 2013; Freire et al., 2014a; Llibre \& Ponce, 2012; Llibre et al., 2013], it took some time to find the first example with two limit cycles and it even was erroneously conjectured in 2010 that two would be the maximum number of limit cycles for this family of piecewise linear differential systems, see [Han \& Zhang, 2010]. It is worth observing that our proof of statement (v) gives a very easy way of finding examples of systems with exactly two hyperbolic crossing limit cycles. In fact, an advantage of the examples that we present is that the total algebraization of the problem allows to prove in many cases that the found limit cycles are the only periodic solutions of the system.

It is also interesting to notice that all examples with three limit cycles have at least one of the two linear systems with a (real or virtual) equilibrium point of focus type. Therefore, in view of item (i) it is quite natural that we have found "only" two algebraic limit cycles. This suggests the following natural question:

Question: Are there piecewise linear differential systems of type (2) with three or more algebraic limit cycles?

All our attempts to answer this question failed. We have also tried to find triple algebraic limit cycles without success, see Remark 2.1 for more detailed comments.

It also worthwhile to observe that there is no explicit quadratic system having a double limit cycle, those are only existential examples which are provided in the literature. On the other hand, in the piecewise linear differential setting we have been able to give such explicit examples, see Propositions 4.1 and 4.2.

In Subsection 2.2 we give an example of algebraic limit cycle with sliding (that is, not of crossing type) of a nonlinear piecewise differential systems, showing that the linearity assumption is needed to prove statements (ii) and (iv). Obviously, the result of item (iii) is also exclusive to linear systems of type (2).

Finally notice, that in contrast with what happens for quadratic systems, where the highest degree of known algebraic limit cycles is 6 , in our context we have totally solved the question about the allowed degrees of the algebraic limit cycles and there is no restriction for them.

This paper is structured as follows. Section 2 describes which are the type of systems that we are dealing with, proves items (i) to (iv) of Theorem 1.1 and also gives some preliminary results. The method 
used to analyze the stability and hyperbolicity of the periodic orbits is developed in Subsection 2.1 and an example of algebraic limit cycle with sliding for a nonlinear system is given in Subsection 2.2. Examples with exactly two hyperbolic algebraic limit cycles proving statement (v) are given in Section 3 . A bifurcation of saddle-node of algebraic limit cycles inside the piecewise linear differential systems class is presented in Section 4. Finally, in Section 5 we show the existence of algebraic limit cycles of degree $(m, n)$ for arbitrary $m$ and $n$, greater than or equal to two, proving statement (vii).

\section{Systems with algebraic level curves}

This section is devoted to prove statements (i) to (iv) of Theorem 1.1. We also give an example of sliding limit cycle for a piecewise nonlinear differential system and introduce the approach that we will use to study the stability of the algebraic limit cycles.

Proof. $\quad$ Proof of statement (i)] Because the vector fields $X^{ \pm}$are linear, it is clear that the isolated periodic orbits of system (2) must cross $\Sigma$. As we have commented in the introduction, continua of periodic orbits, corresponding to the invariant ellipses associated to a real center, might lie in any of the regions $\Sigma^{ \pm}$, but limit cycles are never present.

Notice first that the algebraic pieces of any periodic orbit in $\Sigma^{ \pm}$can not be formed with pieces of invariant straight lines. This statement follows once we prove that if a smooth linear system has an irreducible invariant algebraic curve of degree bigger than 1, then it is of one of the following two types:

- A linear system with a unique equilibrium point of type center, saddle, or node with different eigenvalues, and such that the quotient among the corresponding eigenvalues is a nonzero rational number.

- A linear system without equilibrium points.

To prove the above assertion we assume first that one of the vector fields, say $X^{+}$but extended to the whole plane, has some equilibrium point. If this point is not isolated, then the linear system writes as $\left(x^{\prime}, y^{\prime}\right)=((a x+b y+c) k,(a x+b y+c) \ell)$ for some real constants $a, b, c, k$, and $\ell$. Hence it has no invariant algebraic curve of degree bigger than 1 . Thus, we can assume that the determinant of differential of $X^{+}$ at the equilibrium points is not zero.

We study separately the cases with 0,1 or 2 real invariant straight lines passing through the equilibrium point. Is clear that, in the first case, the point is a focus or a center. The systems with focus points can not have algebraic curves and, in contrast, the systems with centers have all their invariant curves algebraic (are ellipses). In the second case, from the explicit solutions, the level sets, different from straight lines, are all non algebraic. In the last case, after an affine change of variables if necessary, the system writes as $\left(u^{\prime}, v^{\prime}\right)=\left(\lambda_{1} u, \lambda_{2} v\right)$ where $\lambda_{1}$ and $\lambda_{2}$ are the eigenvalues. In these coordinates the system is integrable and a first integral writes as $|u|^{\lambda_{2}}|v|^{-\lambda_{1}}$. In the original coordinates this first integral is $H_{1}=\left|\ell_{1}\right|^{\lambda_{1}}\left|\ell_{2}\right|^{-\lambda_{2}}$, where the straight lines $\ell_{1}=0$ and $\ell_{2}=0$, which are invariant by the flow, coincide with the eigendirections. The proof in this last case finishes because only when the eigenvalues have rational quotient the solutions are contained in algebraic sets. This is so because if $-\lambda_{1} / \lambda_{2}=p / q$, with $p, q \in \mathbb{Z}$, then $H_{2}=H_{1}^{q / \lambda_{2}}=\left|\ell_{1}\right|^{p}\left|\ell_{2}\right|^{q}$. As $p, q$ are integer numbers,

$$
H(x, y)=\ell_{1}^{p} \ell_{2}^{q}=\left(a_{0} x+a_{1} y+a_{2}\right)^{p}\left(b_{0} x+b_{1} y+b_{2}\right)^{q}
$$

is also a first integral and all its level sets are algebraic curves.

If the vector field, considered again in the whole plane, has no equilibrium points, it can be written as $\left(u^{\prime}, v^{\prime}\right)=((A u+B v+C) D+E,(A u+B v+C))$, for some real parameters $A, B, C, D$, and $E \neq 0$. With the linear change $(x, y)=((u-D v) / E, v)$ it is transformed to

$$
x^{\prime}=1, \quad y^{\prime}=a x+b y+c .
$$

If $b \neq 0$, its only algebraic invariant curve is an invariant line. If $b=0$, it has the first integral

$$
H(x, y)=y-\frac{a}{2} x^{2}-c x
$$

and all its solutions are algebraic and given by parabolas if $a \neq 0$ and by straight lines otherwise. Hence statement (i) follows. 

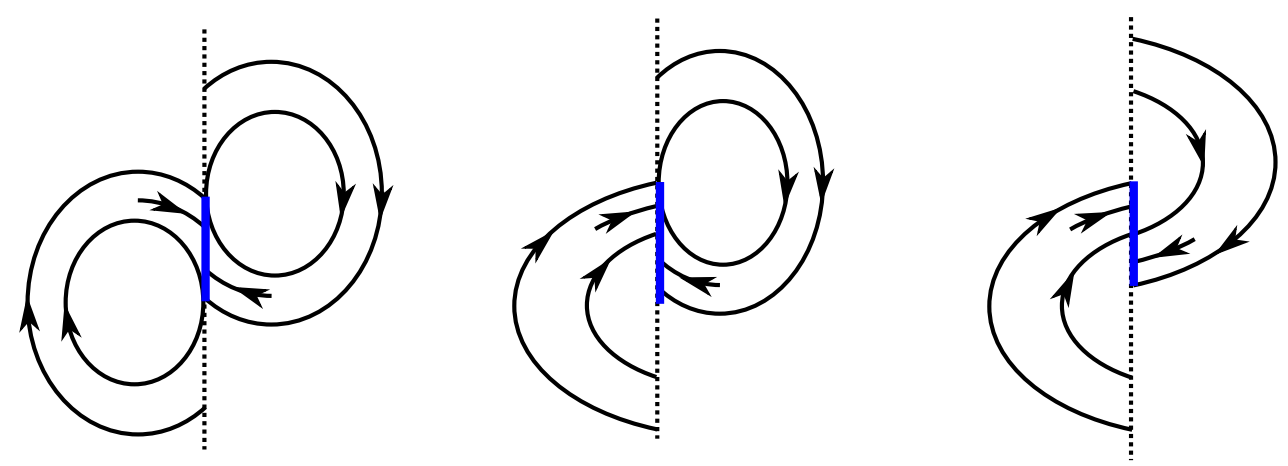

Fig. 2. Different type of repelling sliding segments
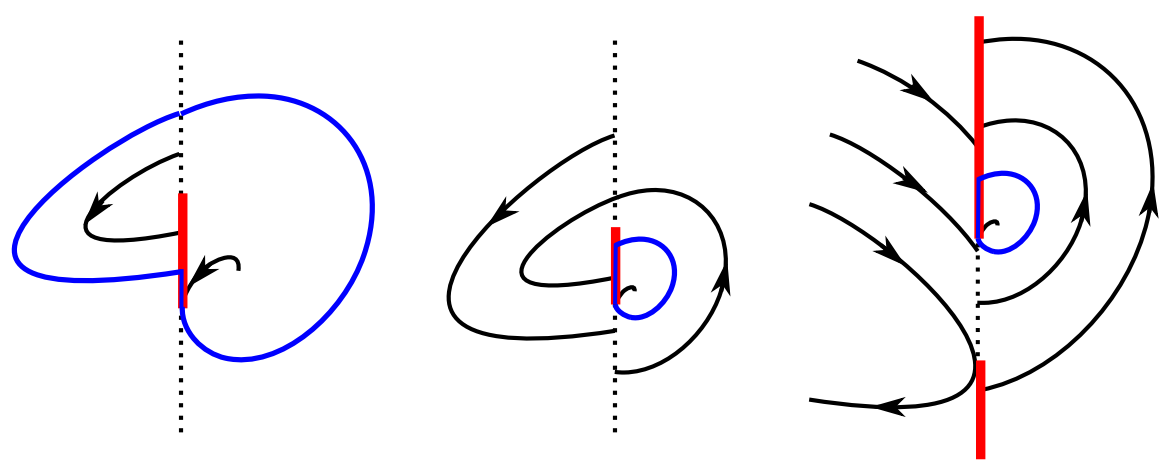

Fig. 3. Different type of sliding limit cycles in the focus case

Proof. [Proof of statement (ii)] Assume that $\Gamma$ is an algebraic limit cycle having a segment $\overline{p q}$ contained in the sliding set. Without loss of generality we can assume that the sliding set is attracting, see Figure 2. The unique way of a trajectory let out the sliding set is via a visible tangency in $\Sigma^{+}$, for example. See the three pictures in Figure 3. A similar case by case study as the one developed in (i) shows that the unique situation such that the trajectory $\Gamma$ come back to $\Sigma$ is when $X^{+}$is of center type. Then, the points $p$ and $q$ coincide and, with our definition, $\Gamma$ is not a limit cycle. This can be observed in Figure 2 (left or center).

Finally, we observe that sliding periodic orbits are only possible if there is a real focus responsible of the visible tangency. See again Figure 3.

Proof. [Proof of statements (iii)] The first part is a direct consequence of the statement (i). This is so because when the system has an algebraic limit cycle indeed all solutions of (2) are formed by (finitely or infinitely many) pieces of algebraic curves. To prove that all limit cycles are nested we proceed by contradiction. Notice that, by statement (ii), all limit cycles are of crossing type. If there are two non nested limit cycles there exists a (non invariant) straight line, $a x+b y+c=0$, tangent to both limit cycles and the tangent points are both in $\Sigma^{+}$. These tangent points are solutions of the linear system $\left\{a x+b y+c=0, a f^{+}(x, y)+b g^{+}(x, y)=0\right\}$. This system has exactly one solution because the straight line is not invariant. This fact contradicts the existence of both tangent points.

Proof. [Proof of statement (iv)] If the algebraic limit cycle is of the form $\Gamma=\{p, p\}$, then $p(x, y)=0$ is a level set of degree bigger than one of one of the algebraic first integrals of the linear systems. Recall that these first integrals are parabolas, ellipses or curves of the form (3). Moreover this level set must be a closed curve. Hence the unique possibility is that $p(x, y)=0$ is an ellipse. Since both systems have the same ellipse as a solution it holds that $X^{+}=\mu X^{-}$for some $\mu \in \mathbb{R} \backslash\{0\}$. In particular the curve $p(x, y)=0$ 
forms part of a continuum of invariant ellipses, so it is an algebraic periodic orbit but it is not a limit cycle, as we wanted to prove.

\subsection{Stability of the crossing algebraic periodic orbits}

The crossing periodic orbits are defined gluing the respective pieces in $\Sigma^{ \pm}$of the two level curves $\left\{H^{+}(x, y)=h^{+}\right\}$and $\left\{H^{-}(x, y)=h^{-}\right\}$that coincide on $\Sigma$ only at the points $(0, u)$ and $(0, v)$. These levels curves are either of the form (3), or ellipses, or parabolas. In particular we can think that $v$ is the half return map of $u$, or viceversa. As $u \neq v$, the functions

$$
C^{ \pm}(u, v)=\left(H^{ \pm}(0, u)-H^{ \pm}(0, v)\right) /(u-v) .
$$

are well defined. Then, the curves $C^{ \pm}=0$, in the $(u, v)$-plane, define implicitly the considered half return maps in $\Sigma^{ \pm}$. Consequently, the periodic solutions are in one to one correspondence with the intersection points of the curves $\left\{C^{+}(u, v)=0\right\}$ and $\left\{C^{-}(u, v)=0\right\}$. We remark that the system is symmetric in the variables $(u, v)$ because we have not fixed where $u$ and $v$ are located in the $y$-axis. We can restrict the solutions to the ones satisfying $u<v$. We have removed, from $H^{ \pm}(0, u)-H^{ \pm}(0, v)$ the factor $u-v$ because the trivial solution $v=u$ gives no information.

The stability of the periodic orbits can be also studied from the intersections of the curves $\left\{C^{+}(u, v)=\right.$ $0\}$ and $\left\{C^{-}(u, v)=0\right\}$. Let $\left(u_{0}, v_{0}\right)$ be an intersection point. The half return maps in $\Sigma^{ \pm}$near the corresponding periodic orbit follow from the series expansion at $u=u_{0}$ of the function $v^{ \pm}(u)$ defined implicitly by $C^{ \pm}(u, v(u))=0$, as we have explained before. As $u_{0}$ coincides in both half return maps, we will use the difference map, $v^{+}(u)-v^{-}(u)$, instead of the composition of them. Then, the zeros of the difference map provide the crossing periodic orbits.

Writing the half return maps as

$$
v^{ \pm}(u)=v_{0}+V_{1}^{ \pm}\left(u-u_{0}\right)+V_{2}^{ \pm}\left(u-u_{0}\right)^{2}+V_{3}^{ \pm}\left(u-u_{0}\right)^{3}+\cdots,
$$

the difference map is

$$
v^{+}(u)-v^{-}(u)=L_{1}\left(u-u_{0}\right)+L_{2}\left(u-u_{0}\right)^{2}+L_{3}\left(u-u_{0}\right)^{3}+\cdots,
$$

where $L_{i}=V_{i}^{+}-V_{i}^{-}$for $i=1,2,3, \ldots$

The first non zero $L_{i}$ provides the stability inside and outside the periodic orbit, otherwise we have a center. In this case we say that $i$ is the multiplicity of the periodic orbit. Moreover, if $L_{1}$ is different from zero the periodic orbit is a hyperbolic limit cycle. The attractor or repeller character of a limit cycle can be determined using the rotation direction and the sign of $L_{1}$. When $L_{1}$ vanishes and $L_{2}$ not we say that the limit cycle is double. In this last case it is semistable. Examples of both situations together with a saddle-node bifurcation of periodic orbits are showed in the next sections.

This notion coincides with the stability criteria obtained using the first non vanishing derivative of the composition of both return maps in $\Sigma^{ \pm}$at the fixed point.

Remark 2.1. In all our computations with piecewise linear differential systems having algebraic level curves such that $L_{1}=L_{2}=0$ and $L_{3} \neq 0$ the intersection points do not correspond to limit cycles because at least one of the invariant straight lines crosses $\Sigma$ in the segment formed by the points $\left(0, u_{0}\right)$ and $\left(0, v_{0}\right)$. Hence, we have found no limit cycle of multiplicity three. Additionally, in all cases we have tried, when $L_{1}=L_{2}=L_{3}=0$ always $L_{4}=L_{5}=0$.

\subsection{An algebraic limit cycle with sliding}

We have proved in statement (ii) that all algebraic limit cycles for linear piecewise differential systems have no sliding. This section is devoted to show that removing this linearity assumption in one of the vector fields $X^{ \pm}$this type of algebraic limit cycles can exist. In fact, we introduce a one-parameter family of piecewise polynomial differential systems $X^{ \pm}$with degrees 1 and 2 having an algebraic limit cycle that passes from being of crossing type to being of sliding type. In particular the family presents a critical crossing cycle bifurcation, see [Freire et al., 2015] for more details on this bifurcation. Recently, in [Novaes et al., 2018] this bifurcation also appears but without studying if the limit cycles are or not algebraic. 
Proposition 2.2. The system

$$
\begin{aligned}
& X^{+}:\left(x^{\prime}, y^{\prime}\right)=\left(-3 y^{2}+8 y-4,-1\right), \\
& X^{-}:\left(x^{\prime}, y^{\prime}\right)=(y-a,-x),
\end{aligned}
$$

defined in $\Sigma^{ \pm}=\left\{(x, y) \in \mathbb{R}^{2}: \pm x \geq 0\right\}$, has a unique limit cycle when $2 / 3<a<2$, otherwise the system has no periodic solutions. This limit cycle is algebraic of degree $(2,3)$ and writes as

$$
\Gamma_{1}=\left\{x^{2}+(y-a)^{2}+3 a^{2}-8 a+4, x-4 y+4 y^{2}-y^{3}+16-40 a+32 a^{2}-8 a^{3}\right\},
$$

when $2 / 3<a \leq 1$, and

$$
\Gamma_{2}=\left\{x^{2}+(y-a)^{2}-a^{2}, x-4 y+4 y^{2}-y^{3}\right\}
$$

when $1 \leq a<2$. Moreover, when $2 / 3<a<1$ it is of crossing type and stable and when $1 \leq a<2$ it intersects the sliding segment.

In Figure 4 we show the evolution of the phase portrait between the bifurcation values $a=2 / 3$ and $a=2$. For the special value $a=1$, one of the intersection points of the limit cycle with the separation line is a tangent point.
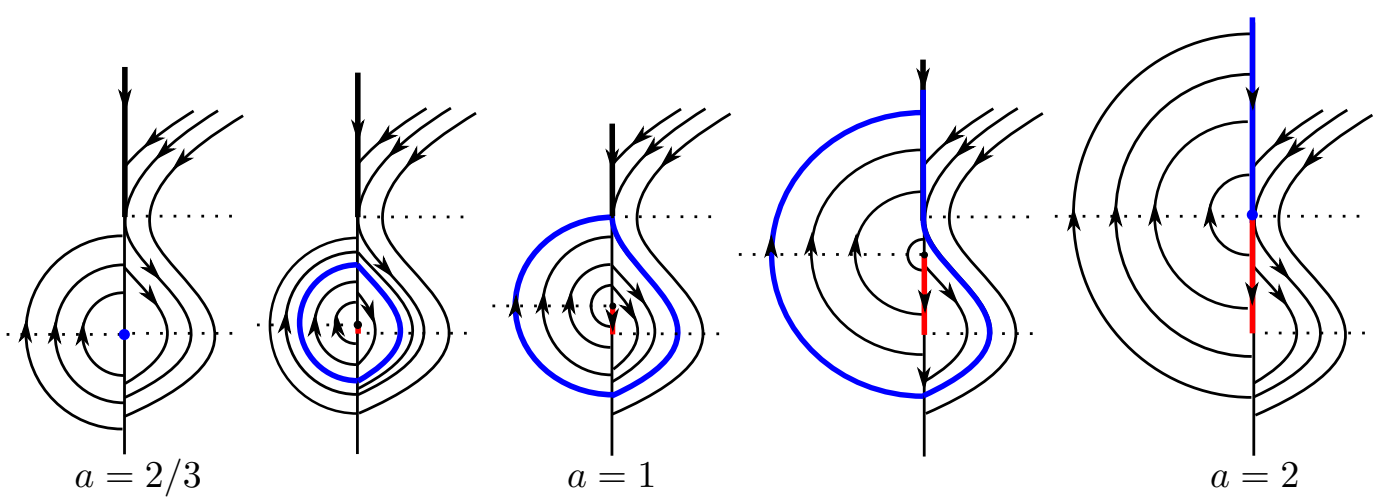

Fig. 4. One-parameter family with an algebraic limit cycle that changes from crossing $(2 / 3<a<1)$ to sliding $(1<a<2)$ type

Proof. [Proof of Proposition 2.2] Straightforward computations show that all the solutions of (5) are included in the level curves of the polynomials $H^{ \pm}$with $H^{-}(x, y)=x^{2}+(y-a)^{2}$ and $H^{+}(x, y)=x-4 y+$ $4 y^{2}-y^{3}$. Moreover, the sliding region is defined by the two segments $(\max \{a, 2\}, \infty)$ and $(2 / 3, \min \{a, 2\})$, when $a>2 / 3$, or $(a, 2 / 3)$, when $a<2 / 3$. The first is an attractor and the second is a repeller. It is easy to see that periodic orbits can only exist when $2 / 3<a<2$, and in fact, the crossing and sliding limit cycles exist when $2 / 3<a<1$ and $1 \leq a<2$, respectively. From the phase portraits depicted in Figure 4 is clear that the sliding limit cycle is unique. The proof finishes using the method described in Section 2.1, checking that, for $a \in(2 / 3,1)$, there is only one crossing limit cycle and it is hyperbolic and attractor.

The uniqueness follows because the curves (4),

$$
C^{+}=-u^{2}-u v-v^{2}+4 u+4 v-4, \quad C^{-}=u+v-2 a,
$$

have only one intersection point satisfying $u<v$. In particular

$$
u=a-\sqrt{(3 a-2)(2-a)}, \quad v=a+\sqrt{(3 a-2)(2-a)} .
$$

Finally, we can obtain its stability,

$$
L_{1}=\frac{-4 \sqrt{(3 a-2)(2-a)}(2-a)}{(\sqrt{(3 a-2)(2-a)}+2-a)(\sqrt{(3 a-2)(2-a)}+3 a-6)} .
$$




\section{Statement (v): Two explicit algebraic hyperbolic limit cycles}

In [Llibre \& Zhang, 2016] it is proved that the maximum number of limit cycles (not necessarily algebraic) for piecewise linear differential systems is two when in one side the system has a center and that it is one if the other system has not a focus point. Since in statement (v) we are interested on having at least two limit cycles, in our examples for proving this statement (and also statement (vi)) we do not consider the case of one of the systems having a center. We notice that it is not difficult to find examples with a unique algebraic and hyperbolic limit cycle with one of the equilibrium points of the associated smooth linear systems of center type, see for instance system (1) in the introduction. For similar reasons we do not consider systems that have, in one side, a system without equilibrium points.

The next two results provide examples of piecewise linear differential systems having two explicit algebraic and hyperbolic limit cycles. The first has two real saddles and the second two virtual nodes. Figure 5 shows the drawing of the periodic solutions and the invariant straight lines in both cases.
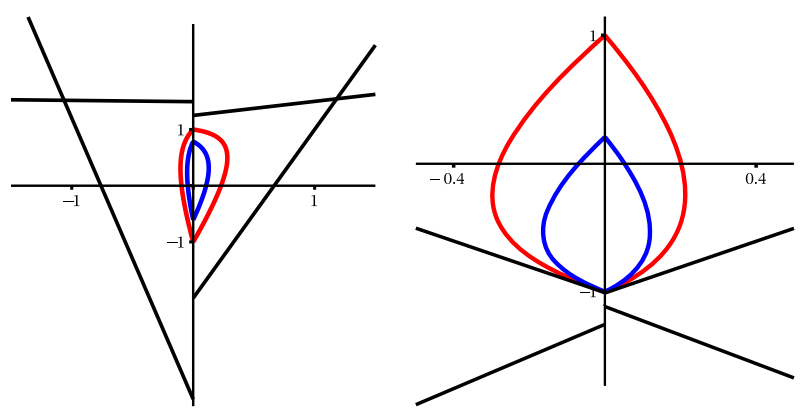

Fig. 5. Piecewise linear differential systems with two algebraic hyperbolic limit cycles

Proposition 3.1. Let $a$ be the smallest root of the polynomial $p(z)=4704 z^{4}+17248 z^{3}-2385 z^{2}+773$, $a \approx-3.797189105$. The piecewise linear differential system

$$
\begin{aligned}
& X^{+}:\left(x^{\prime}, y^{\prime}\right)=(14 x-12 y+2,9 x-25 y+28), \\
& X^{-}:\left(x^{\prime}, y^{\prime}\right)=\left(-\left(15 a^{2}+8\right) x-\left(12 a^{2}+4\right) y-8 a, 20 x+\left(45 a^{2}+16\right) y+a\left(15 a^{2}+44\right)\right),
\end{aligned}
$$

defined in $\Sigma^{ \pm}=\left\{(x, y) \in \mathbb{R}^{2}: \pm x \geq 0\right\}$ has exactly two limit cycles. One crosses the $y$-axis at $(0,-1)$ and $(0,1)$, and the other at $(0,-17 / 28)(0,11 / 14)$. Moreover, they are algebraic, hyperbolic and have opposite stability, and they write as $\left\{H^{-}(x, y)-\left(a^{2}-1\right)^{3}, H^{+}(x, y)-9\right\}$ and $\left\{H^{-}(x, y)-(28 a+17)^{3}\left(28 a^{3}-51 a^{2}+\right.\right.$ $\left.84 a-17) / 614656, H^{+}(x, y)-19773 / 1372\right\}$ with

$$
\begin{aligned}
& H^{+}(x, y)=(x-4 y+5)(-3 x+y+2)^{2}, \\
& H^{-}(x, y)=\left(x+\left(3 a^{2}+1\right) y+a\left(a^{2}+3\right)\right)(a-5 x-y)^{3} .
\end{aligned}
$$

Moreover, these two limit cycles surround a sliding segment.

Proof. First we will prove that system (7) has two periodic solutions described by the level sets of the statement. The second step will be the proof that no other periodic orbit exists. Finally, we show the hyperbolicity and stability properties.

Systems defined in (7) have two saddle equilibrium points in the intersection point of two invariant straight lines that cross $y$-axis in $(0,5 / 4),(0,-2)$ for $X^{+}$and $(0, a),\left(0,-a\left(a^{2}+3\right) /\left(3 a^{2}+1\right)\right)$ for $X^{-}$. Then, the solutions of (6) can be obtained gluing pieces of hyperbolas like curves defined by the level sets $H^{ \pm}=h^{ \pm}$varying $h^{ \pm}$, where $H^{ \pm}$are defined in $(7)$. Both piecewise algebraic curves are periodic orbits only if they are connex in the respective half planes where they are defined and they do not contain any real equilibrium point. Consequently, since $a<0$, the crossing points of the periodic orbits with the $y$-axis should be between $(0, \max (-2,-a))$ and $\left(0, \min \left(5 / 4,-a\left(a^{2}+3\right) /\left(3 a^{2}+1\right)\right)\right.$. 
Straightforward computations show that the solutions passing through the crossing points $(0,-1)$ and $(0,-17 / 28)$ correspond to the levels defined in the statement. Additionally, these curves pass also through the crossing points $(0,1)$ and $(0,11 / 14)$, respectively.

For system (6), the two limit cycles appear as it is shown in the left-hand picture of Figure 5 . The polynomial $p(z)$ has another real root $b>a$. Changing $a$ by $b \approx-0.3220697181$ in system (6), it has no limit cycle, see Figure 6 . Observe that only for the smallest root the pieces of the hyperbolas passing trough the crossing points define a closed curve.

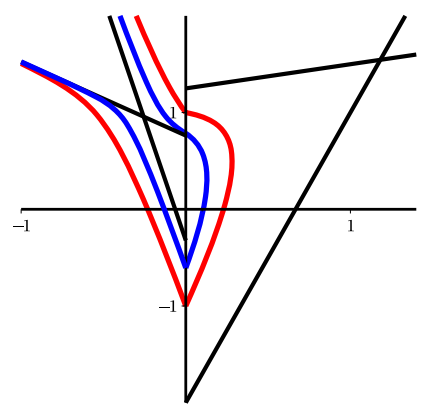

Fig. 6. Phase portrait of system (6) changing $a$ by $b$

Using the method described in Section 2, the non existence of other periodic solutions can be proved studying the intersection points of the curves $C^{ \pm}=0$ in (4) that, for system (6), write as

$$
\begin{aligned}
C^{+}(u, v)= & -4 u^{2}-4 v u-4 v^{2}-11 u-11 v+4=0, \\
C^{-}(u, v)= & -\left(3 a^{2}+1\right) u^{3}-\left(3 a^{2}+1\right) v u^{2}-\left(3 a^{2}+1\right) u v^{2}-\left(3 a^{2}+1\right) v^{3} \\
& +8 a^{3} u^{2}+8 a^{3} u v+8 a^{3} v^{2}-6 a^{2}\left(a^{2}-1\right) u-6 a^{2}\left(a^{2}-1\right) v-8 a^{3}=0 .
\end{aligned}
$$

The above system of equations, with the symmetry restriction and taking into account that $a$ satisfies $p(a)=0$, has only two real solutions $(1,-1)$ and $(-17 / 28,11 / 14)$. This can be proved because, modulus some non zero rational constants and the factor $51744 a^{3}-16563 a^{2}+751$, the resultant of $C^{+}(u, v)$ and $C^{-}(u, v)$ with respect to $v$, simplified taking into account that $p(a)=0$, is

$$
D(u):=\left(u^{2}-1\right)(14 u-11)(28 u+17)\left(392 u^{2}+2226 u+6127\right),
$$

and the one respect to $u$ is, by symmetry, $D(v)=0$, and it is well known that the real and complex solutions of the system $\left\{C^{+}(u, v)=0, C^{-}(u, v)=0\right\}$ are contained into the ones of the uncoupled system $\{D(u)=0, D(v)=0\}$. The twelve real spurious solutions provided by the method can simply be discarded replacing them into the initial equations $C^{ \pm}(u, v)=0$.

Only remains to prove the hyperbolicity condition. This can be done also using the curves $C^{ \pm}$. In fact, we only need to compute the series expansion of each piecewise continuous solution $v(u)$ near $u=-1$ and $u=-17 / 28$ for both $C^{ \pm}$. Straightforward computations show that

$$
\begin{aligned}
& v^{+}(u)=1-\frac{7}{15}(u+1)-\frac{676}{3375}(u+1)^{2}+\cdots, \\
& v^{-}(u)=1-\frac{10976 a^{3}-2385 a^{2}-795}{15\left(1568 a^{3}-159 a^{2}-53\right)}(u+1)+\cdots, \\
& v^{+}(u)=\frac{11}{14}-\frac{5}{8}\left(u+\frac{17}{28}\right)-\frac{343}{1664}\left(u+\frac{17}{28}\right)^{2}+\cdots, \\
& v^{-}(u)=\frac{11}{14}-\frac{5\left(1456 a^{3}+51 a^{2}+17\right)}{2\left(5824 a^{3}-165 a^{2}-55\right)}\left(u+\frac{17}{28}\right)+\cdots,
\end{aligned}
$$


and the differences give the hyperbolicity because the coefficients, which are different from zero, of degree 1 on $(u+1)$ and $(u+17 / 28)$ are respectively

$$
-\frac{424\left(3 a^{2}+1\right)}{15\left(1568 a^{3}-159 a^{2}-53\right)} \quad \text { and } \quad \frac{615\left(3 a^{2}+1\right)}{8\left(5824 a^{3}-165 a^{2}-55\right)} .
$$

Moreover, as the product of the above numbers is negative, the limit cycles have opposite stability.

Furthermore, notice that system (6) has two tangent points of $X^{ \pm}$that define, over $\Sigma$, the sliding segment $\left(1 / 6,-2 a /\left(3 a^{2}+1\right)\right)$, that it is inside the smallest periodic orbit.

Similarly as the previous proof, the next proposition, exhibiting two explicit algebraic hyperbolic limit cycles in the node-node case, follows. For this new system the first integrals are $H^{ \pm}(x, y)=$ $P^{ \pm}(x, y) / Q^{ \pm}(x, y)$ and the level curves which define the limit cycles are $\left\{H^{+}(x, y)=-1\right\},\left\{H^{+}(x, y)=\right.$ $-736 / 475\},\left\{H^{-}(x, y)=-1\right\}$, and $\left\{H^{-}(x, y)=-19044 / 8575\right\}$, where

$$
\begin{array}{ll}
P^{+}(x, y)=(181 x-180 y-181), & Q^{+}(x, y)=(10 x+9 y+10)^{2}, \\
P^{-}(x, y)=(365 x+364 y+365), & Q^{-}(x, y)=(5 x-4 y-5)^{3} .
\end{array}
$$

Proposition 3.2. The piecewise linear differential system

$$
\begin{aligned}
& X^{+}:\left(x^{\prime}, y^{\prime}\right)=(5058 x-1620 y-1458,-1810 x+5229 y+5430), \\
& X^{-}:\left(x^{\prime}, y^{\prime}\right)=(-3100 x-1456 y-1280,-1825 x-3460 y-3650),
\end{aligned}
$$

defined in $\Sigma^{ \pm}=\left\{(x, y) \in \mathbb{R}^{2}: \pm x \geq 0\right\}$ has exactly two limit cycles, one crossing the $y$-axis at $(0,-1)$ and $(0,1)$, and another at $(0,-275 / 276)$ and $(0,5 / 24)$. Moreover, they are algebraic and hyperbolic, and are $\left\{P^{-}(x, y)+Q^{-}(x, y), P^{+}(x, y)+Q^{+}(x, y)\right\}$ and $\left\{8575 P^{-}(x, y)+19044 Q^{-}(x, y), 475 P^{+}(x, y)+736 Q^{+}(x, y)\right\}$.

It is not difficult to find simpler piecewise linear differential systems having two algebraic limit cycles. This can be done, for instance, using the normal forms introduced in [Freire et al., 2014b]. Nevertheless, it is not easy to find rational initial conditions on $\Sigma$ for both limit cycles.

\section{Statement (vi): Double algebraic limit cycles}

This section is devoted to show the existence of explicit double (semistable) algebraic limit cycles in the saddle-node and saddle-saddle cases. We only give the details in the first case, because both proofs are similar. The periodic orbits for both systems together with the corresponding invariant straight lines are drawn in Figure 7.
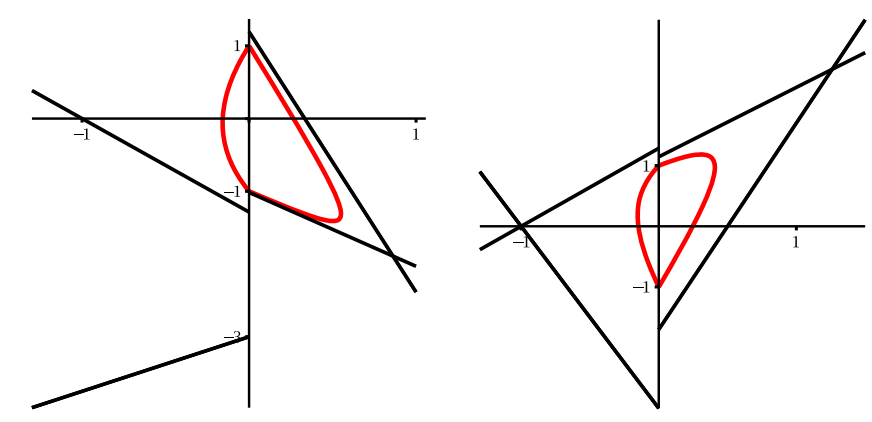

Fig. 7. Piecewise linear differential systems with an algebraic double limit cycle 
Proposition 4.1. The piecewise linear differential system

$$
\begin{aligned}
X^{+}: x^{\prime} & =(236171-25888 \sqrt{889}) x-(465183-5994 \sqrt{889}) y+344063-14224 \sqrt{889} \\
y^{\prime} & =1041255 x+(3293+26066 \sqrt{889}) y+578475 \\
X^{-}: x^{\prime} & =-(129-324 \varepsilon) x-(56-168 \varepsilon) y-24+324 \varepsilon \\
y^{\prime} & =-54 x-(99-108 \varepsilon) y-189
\end{aligned}
$$

defined in $\Sigma^{ \pm}=\left\{(x, y) \in \mathbb{R}^{2}: \pm x \geq 0\right\}$ has, for $\varepsilon$ small enough, exactly 0, 1 double, or 2 hyperbolic limit cycles with opposite stability when $\varepsilon<0, \varepsilon=0$, or $\varepsilon>0$, respectively. When $\varepsilon=0$, the limit cycle crosses the $y$-axis at $(0,-1)$ and $(0,1)$ and it is algebraic. Moreover, it writes as $\left\{256 P^{-}(x, y)+\right.$ $\left.Q^{-}(x, y), H^{+}(x, y)-15854096+526288 \sqrt{889}\right\}$ with

$$
\begin{aligned}
& H^{+}(x, y)=(4285 x+(999+108 \sqrt{889}) y+4285)(81 x-(37-2 \sqrt{889}) y-27)^{2} \\
& H^{-}(x, y)=\frac{P^{-}(x, y)}{Q^{-}(x, y)}=\frac{9 x+7 y+9}{(3 x-4 y-12)^{3}} .
\end{aligned}
$$

Proof. It is easy to see that the solutions of (8) are defined by the level sets of $H^{+}$given in (9) and

$$
H_{\varepsilon}^{-}(x, y)=(9 x+7 y+9)(3 x-(4-12 \varepsilon) y-12)^{-3} \text {. }
$$

This, taking $\varepsilon=0$, proves the last statement.

Using the method described in Section 2 the periodic orbits can be determined from the intersection of the curves $C^{ \pm}(u, v)=0$ defined in (4). Consequently, for every $\varepsilon$, the limit cycles for equation (8) are the intersection of the two curves

$$
\begin{aligned}
C^{+}(u, v)= & 27 u^{2}+27 u v+27 v^{2}-37 u-37 v-27=0, \\
C_{\varepsilon}^{-}(u, v)= & -7(1-3 \varepsilon)^{3} u^{2} v-7(1-3 \varepsilon)^{3} u v^{2}-9(1-3 \varepsilon)^{3} u^{2}-9(8-3 \varepsilon)(1-3 \varepsilon)^{2} u v \\
& -9(1-3 \varepsilon)^{3} v^{2}-81(1-3 \varepsilon)^{2} u-81(1-3 \varepsilon)^{2} v-54+729 \varepsilon=0 .
\end{aligned}
$$

In the above functions we have removed some non zero factors. We recall that they define implicitly the first return map. The resultant of $C^{+}$and $C_{\varepsilon}^{-}$with respect to $v$ is

$$
D(u, \varepsilon)=\operatorname{res}\left(C^{+}, C_{\varepsilon}^{-}, v\right)=1728\left(-1400 \varepsilon+5047(u+1)^{2}-49224 \varepsilon(u+1)+86637 \varepsilon^{2}\right)+O_{3}(u+1, \varepsilon) .
$$

By using the Newton diagram, the local expansions of the branches of the curve $D=0$ near $(u, \varepsilon)=(-1,0)$ are given by its principal part. They can be obtained solving $-1400 \varepsilon+5047(u+1)^{2}=0$. Notice that this equation has no solution for $\varepsilon<0$ and two simple solutions when $\varepsilon>0$. Associated to each of these two solutions, the series expansion, near $\varepsilon=0$, of the intersection points are

$$
\begin{aligned}
& u_{ \pm}=-1 \pm \frac{10 \sqrt{1442}}{721} \sqrt{\varepsilon}+\frac{371498}{74263} \varepsilon+\cdots, \\
& v_{ \pm}=1 \mp \frac{64 \sqrt{1442}}{721} \sqrt{\varepsilon}-\frac{399740}{74263} \varepsilon+\cdots .
\end{aligned}
$$

The series expansion of $v^{ \pm}(u)$, defined implicitly from $C_{\varepsilon}^{ \pm}$, near $u_{ \pm}$in (10), write as

$$
\begin{aligned}
v^{+}\left(u_{ \pm}\right)= & \left(1 \mp \frac{64}{721} \sqrt{1442} \sqrt{\varepsilon}+\cdots\right)+\left(-\frac{32}{5} \pm \frac{6858}{2575} \sqrt{1442} \sqrt{\varepsilon}+\cdots\right)\left(u-u_{ \pm}\right) \\
& +\left(\frac{24003}{250} \mp \frac{16387191}{128750} \sqrt{1442} \sqrt{\varepsilon}+\cdots\right)\left(u-u_{ \pm}\right)^{2}+\cdots, \\
v^{-}\left(u_{ \pm}\right)= & \left(1 \mp \frac{64}{721} \sqrt{1442} \sqrt{\varepsilon}+\cdots\right)+\left(-\frac{32}{5} \pm \frac{1296}{2575} \sqrt{1442} \sqrt{\varepsilon}+\cdots\right)\left(u-u_{ \pm}\right) \\
& +\left(\frac{2268}{125} \mp \frac{145152}{64375} \sqrt{1442} \sqrt{\varepsilon}+\cdots\right)\left(u-u_{ \pm}\right)^{2}+\cdots
\end{aligned}
$$


The proof finishes studying the stability of the periodic orbits computing the difference between the previous expressions,

$$
\begin{aligned}
v^{+}\left(u_{ \pm}\right)-v^{-}\left(u_{ \pm}\right)= & \left( \pm \frac{54}{25} \sqrt{1442} \sqrt{\varepsilon}+\cdots\right)\left(u-u_{ \pm}\right)+ \\
& \left(\frac{19467}{250} \mp \frac{16096887}{128750} \sqrt{1442} \sqrt{\varepsilon}+\cdots\right)\left(u-u_{ \pm}\right)^{2}+\cdots
\end{aligned}
$$

Clearly, when $\varepsilon>0$ the two limit cycles are hyperbolic with opposite stability and when $\varepsilon=0$ the limit cycle has multiplicity 2 , that is double and semistable.

Proposition 4.2. The piecewise linear differential system

$$
\begin{aligned}
X^{+}: x^{\prime} & =(-720776+32380 \sqrt{1477}) x-(655695-9882 \sqrt{1477}) y-854183+23632 \sqrt{1477}, \\
y^{\prime} & =1075194 x+(226981-24938 \sqrt{1477}) y+537597, \\
X^{-}: x^{\prime} & =(3-81 \varepsilon) x-(14-84 \varepsilon) y+3-81 \varepsilon, \\
y^{\prime} & =-54 x+(27+27 \varepsilon) y-54,
\end{aligned}
$$

defined in $\Sigma^{ \pm}=\left\{(x, y) \in \mathbb{R}^{2}: \pm x \geq 0\right\}$ has, for $\varepsilon$ small enough, exactly 0, 1 double, or 2 hyperbolic limit cycles with opposite stability when $\varepsilon<0, \varepsilon=0$, or $\varepsilon>0$, respectively. When $\varepsilon=0$, it crosses the $y$-axis at $(0,-1)$ and $(0,1)$ and it is algebraic and double. Moreover, it writes as $\left\{H^{-}(x, y)-128, H^{+}(x, y)-\right.$ $56943536+1441552 \sqrt{1477}\}$ with

$$
\begin{aligned}
& H^{+}(x, y)=(6637 x-(1647+108 \sqrt{1477}) y+6637)(54 x+(61-2 \sqrt{1477}) y-27)^{2}, \\
& H^{-}(x, y)=(9 x-7 y+9)(3 x+y+3)^{3} .
\end{aligned}
$$

Proof. The proof follows as Proposition 4.1. Now $H_{\varepsilon}^{-}(x, y)=(9 x-7 y+9)(3 x+(1-6 \varepsilon) y+3)^{3}$.

\section{Statement (vii): Limit cycles with degree $(m, n)$}

This section is devoted to show explicit piecewise linear differential systems with algebraic limit cycles of arbitrary degree. In the first result the algebraic curves have different degrees and in the second they have the same degree. We remark that the first differential system has a symmetry with respect to $(m, n)$, which implies that, when $m=n$, it has a center. We have found other examples that would allow to treat simultaneously the cases $m \neq n$ and $m=n$, but for them the computations for proving the hyperbolicity of the limit cycle are more involved. So we have decided to split the study in these two cases.

Proposition 5.1. Let $m, n \geq 2$ be different integer numbers. The piecewise linear differential system

$$
\begin{aligned}
X^{+}: x^{\prime} & =n(a-n) x-a(n-1) y+n(a-1), \\
y^{\prime} & =n^{2}(n-1) x+n(a n-1) y, \\
X^{-}: x^{\prime} & =m(b-m) x-b(m-1) y+m(b-1), \\
y^{\prime} & =m^{2}(m-1) x+m(b m-1) y,
\end{aligned}
$$

with $a=\left((n+1)^{n}-(n-1)^{n}\right) /\left((n+1)^{n}+(n-1)^{n}\right)$ and $b=\left((m+1)^{m}-(m-1)^{m}\right) /\left((m+1)^{m}+(m-1)^{m}\right)$, defined in $\Sigma^{ \pm}=\left\{(x, y) \in \mathbb{R}^{2}: \pm x \geq 0\right\}$, has a hyperbolic stable (unstable) algebraic limit cycle of degree $(m, n)$ when $m>n(m<n)$. Moreover, it writes as $\left\{P^{-}(x, y)-h^{-} Q^{-}(x, y), P^{+}(x, y)-h^{+} Q^{+}(x, y)\right\}$ with

$$
\begin{aligned}
& P^{+}(x, y)=n x+a y+1, \quad Q^{+}(x, y)=\left(x+\frac{1}{n} y+1\right)^{n}, \quad h^{+}=\frac{2 n^{n}}{(n+1)^{n}+(n-1)^{n}}, \\
& P^{-}(x, y)=m x+b y+1, \quad Q^{-}(x, y)=\left(x+\frac{1}{m} y+1\right)^{m}, \quad h^{-}=\frac{2 m^{m}}{(m+1)^{m}+(m-1)^{m}} .
\end{aligned}
$$

Proof. The first integrals of the vector fields $X^{ \pm}$are $H^{ \pm}(x, y)=P^{ \pm}(x, y) / Q^{ \pm}(x, y)$. The existence of an algebraic periodic orbit of degree $(m, n)$ follows checking that the level curves $\left\{P^{-}(x, y)-\right.$ 
$\left.h^{-} Q^{-}(x, y), P^{+}(x, y)-h^{+} Q^{+}(x, y)\right\}$ pass through the points $(0, \pm 1)$. The hyperbolicity is proved computing the half return map implicitly from the curves $C^{ \pm}(u, v)=0$ using (4). From the notation of Subsection 2.1 we have that

$$
V_{1}^{+}=-V(n), \quad V_{1}^{-}=-V(m)
$$

with

$$
V(n)=\frac{f(n)-\frac{3 n-1}{n+1}}{\frac{1}{f(n)}+\frac{n-1}{n+1}}, \quad f(n)=\left(\frac{n+1}{n-1}\right)^{n}
$$

and

$$
L_{1}=L_{1}(m, n)=V(m)-V(n) .
$$

The proof follows showing that $V(n)$ is a positive decreasing function for $n \geq 2$. In fact we prove the monotonicity condition extending it to the reals, for $x \geq 4$. Straightforward computations show that $V(2)=9>V(3)=32 / 5>V(4)=25625 / 4617$.

First we prove that $7 \leq f(x) \leq(5 / 3)^{4}$ is a decreasing function for $x \geq 4$. It is easy to check that $f$ satisfies the differential equation $f^{\prime}=f g$ with $g(x)=\log ((x+1) /(x-1))-2 x /\left(x^{2}-1\right)$. Computing $g^{\prime}$ and checking that $g^{\prime}>0$ and $g$ has limit zero at infinity, we get that $g<0$ and $f^{\prime}<0$. So, $f$ is a decreasing function. The inequality follows observing that $f(4)=(5 / 3)^{4}$ and the limit, when $x$ goes to infinity, is $\mathrm{e}^{2}>7$.

The proof finishes proving that the derivative of $V$ is negative and its limit, when $x$ goes to infinity, is $\left(\mathrm{e}^{2}-3\right) /\left(\mathrm{e}^{-2}+1\right)>0$. Straightforward computations show that

$$
V^{\prime}(x)=\frac{\left(x^{2}-1\right) V_{1}(x) f^{\prime}(x)-2 V_{0}(x) f(x)}{(f(x)(x-1)+x+1)^{2}(x-1)^{2}}
$$

with

$$
\begin{aligned}
& V_{1}(x)=(x-1)^{2} f(x)^{2}+2\left(x^{2}-1\right) f(x)-(3 x-1)(x+1), \\
& V_{0}(x)=(x-1)^{2} f(x)^{2}-4 x(x-1) f(x)-(x+1)^{2} .
\end{aligned}
$$

Then, for $x \geq 4$, since $f(x) \geq 7$, we have that

$$
\begin{aligned}
V_{1}(x) & \geq(x-1)^{2} \cdot 7^{2}+2\left(x^{2}-1\right) \cdot 7-(3 x-1)(x+1) \\
& =60 x^{2}-100 x+36>0 .
\end{aligned}
$$

Similarly, since $7 \leq f(x) \leq(5 / 3)^{4}$,

$$
\begin{aligned}
V_{0}(x) & \geq(x-1)^{2} \cdot 7^{2}-4 x(x-1) \cdot\left(\frac{5}{3}\right)^{4}-(x+1)^{2} \\
& =\frac{1388 x^{2}-5600 x+3888}{3^{4}}>0 .
\end{aligned}
$$

Consequently, the numerator of $V^{\prime}$ is negative for $x \geq 4$.

Proposition 5.2. Let $n \geq 2$ be an integer number. The piecewise linear differential system

$$
\begin{aligned}
X^{+}: x^{\prime} & =\left(-n^{2}+2 a\right) x-a(n-1) y+2 a-n, \\
y^{\prime} & =2 n(n-1) x+n(2 a-1) y, \\
X^{-}: x^{\prime} & =\left(-n^{2}+3 b\right) x-b(n-1) y+3 b-n, \\
y^{\prime} & =3 n(n-1) x+n(3 b-1) y,
\end{aligned}
$$


with $a=\left(3^{n}-1\right) /\left(3^{n}+1\right)$ and $b=\left(2^{n}-1\right) /\left(2^{n}+1\right)$, defined in $\Sigma^{ \pm}=\left\{(x, y) \in \mathbb{R}^{2}: \pm x \geq 0\right\}$, has a hyperbolic stable algebraic limit cycle of degree $(n, n)$. Moreover, it writes as $\left\{P^{-}(x, y)-h^{-} Q^{-}(x, y), P^{+}(x, y)-\right.$ $\left.h^{+} Q^{+}(x, y)\right\}$ with

$$
\begin{aligned}
& P^{+}(x, y)=n x+a y+1, \quad Q^{+}(x, y)=\left(x+\frac{1}{2} y+1\right)^{n}, \quad h^{+}=\frac{2^{1+n}}{3^{n}+1}, \\
& P^{-}(x, y)=n x+b y+1, \quad Q^{-}(x, y)=\left(x+\frac{1}{3} y+1\right)^{n}, \quad h^{-}=\frac{3^{n}}{2^{n-1}\left(2^{n}+1\right)} .
\end{aligned}
$$

Proof. The first integrals of the vector fields $X^{ \pm}$are $H^{ \pm}(x, y)=P^{ \pm}(x, y) / Q^{ \pm}(x, y)$. The first steps of the proof are the same as the proof of Proposition 5.1. The main difference is how the hyperbolicity is proved because the expressions of $V_{1}^{ \pm}$, following the method described in Subsection 2.1, are different. From them we write the stability as

$$
L_{1}=L_{1}(n)=-\frac{3^{n}\left(3^{n}-2 n-1\right)}{3^{n-1}(2 n-3)+1}+\frac{2^{n}\left(2^{n}-n-1\right)}{2^{n-1}(n-2)+1} .
$$

The above expression is well defined, for $n \geq 2$, because the denominator of both summands are positive. The numerator writes as

$$
-3^{n-1} 2^{n-1} A_{1}(n)-A_{2}(n)-(n+1) 2^{n},
$$

with

$$
\begin{aligned}
& A_{1}(n)=(n-2) 3^{n+1}-(2 n-3) 2^{n+1}, \\
& A_{2}(n)=9^{n}-n(2 n-7) 6^{n-1}-4^{n}-(2 n+1) 3^{n} .
\end{aligned}
$$

The proof finishes proving that $A_{1}(n)$ and $A_{2}(n)$ are positive for $n \geq 3$, because $L_{1}(2)=-5$. To prove this positiveness, we write

$$
\begin{aligned}
& A_{1}(n)=9(n-2) 2^{n-1}\left(\left(\frac{3}{2}\right)^{n-1}-\frac{8 n-12}{9 n-18}\right), \\
& A_{2}(n)=56^{n-1}\left(\left(\frac{3}{2}\right)^{n-1}-\frac{n(2 n-7)}{5}\right)+4^{n-1}\left(\left(\frac{9}{4}\right)^{n-1}-4\right)+3^{n}\left(3^{n-1}-(2 n+1)\right),
\end{aligned}
$$

where in the last equality we have used $9^{n}=(5+1+3) 9^{n-1}$. Then we can prove, by induction, that the four expressions between brackets of the form $\alpha^{n-1}-\beta(n)$ are positive for $n \geq 3$.

\section{Acknowledgements}

This work has been realized thanks to the MINECO grants MTM2013-40998-P and MTM2016-77278P (FEDER), the AGAUR grants 2014 SGR568 and 2017 SGR1617, the European Community grants FP7-PEOPLE-2012-IRSES 316338 and 318999, and the Brazilian FAPESP grants 2013/24541-0 and 2017/03352-6.

\section{References}

Acary, V., Bonnefon, O. \& Brogliato, B. [2011] Nonsmooth modeling and simulation for switched circuits, Lecture Notes in Electrical Engineering, Vol. 69 (Springer, Dordrecht), ISBN 978-90-481-9680-7, doi: 10.1007/978-90-481-9681-4, URL http://dx.doi.org/10.1007/978-90-481-9681-4.

Brogliato, B. [2016] Nonsmooth mechanics, Third ed., Communications and Control Engineering Series (Springer), ISBN 978-3-319-28662-4; 978-3-319-28664-8, doi:10.1007/978-3-319-28664-8, URL http: //dx.doi.org/10.1007/978-3-319-28664-8.

Buzzi, C., Pessoa, C. \& Torregrosa, J. [2013] "Piecewise linear perturbations of a linear center," Discrete Contin. Dyn. Syst. 33, 3915-3936.

Chavarriga, J., Giacomini, H. \& Llibre, J. [2001] "Uniqueness of algebraic limit cycles for quadratic systems," J. Math. Anal. Appl. 261, 85-99, doi:10.1006/jmaa.2001.7476, URL http://dx.doi.org/10. 1006/jmaa.2001.7476. 
di Bernardo, M., Budd, C. J., Champneys, A. R. \& Kowalczyk, P. [2008] Piecewise-smooth dynamical systems, Applied Mathematical Sciences, Vol. 163 (Springer-Verlag London, Ltd., London), ISBN 978-1-84628-039-9.

Filippov, A. F. [1988] Differential equations with discontinuous righthand sides, Mathematics and its Applications (Soviet Series), Vol. 18 (Kluwer Academic Publishers Group, Dordrecht), ISBN 90-277-2699-X.

Freire, E., Ponce, E. \& Torres, F. [2014a] "The discontinuous matching of two planar linear foci can have three nested crossing limit cycles," Publ. Mat. 58, 221-253, URL http://projecteuclid.org/euclid.pm/ 1400505235.

Freire, E., Ponce, E. \& Torres, F. [2014b] "A general mechanism to generate three limit cycles in planar Filippov systems with two zones," Nonlinear Dynam. 78, 251-263, URL https://doi.org/10.1007/ s11071-014-1437-7.

Freire, E., Ponce, E. \& Torres, F. [2015] "On the critical crossing cycle bifurcation in planar Filippov systems," J. Differential Equations 259, 7086-7107, doi:10.1016/j.jde.2015.08.013, URL http://dx. doi.org/10.1016/j.jde.2015.08.013.

Guardia, M., Seara, T. M. \& Teixeira, M. A. [2011] "Generic bifurcations of low codimension of planar Filippov systems," J. Differential Equations 250, 1967-2023, doi:10.1016/j.jde.2010.11.016, URL http: //dx.doi.org/10.1016/j.jde.2010.11.016.

Han, M. \& Zhang, W. [2010] "On Hopf bifurcation in non-smooth planar systems." J. Differential Equations 248, 2399-2416.

Kunze, M. [2000] Non-smooth dynamical systems, Lecture Notes in Mathematics, Vol. 1744 (SpringerVerlag, Berlin), ISBN 3-540-67993-6, doi:10.1007/BFb0103843, URL http://dx.doi.org/10.1007/ BFb0103843.

Kuznetsov, Y. A., Rinaldi, S. \& Gragnani, A. [2003] "One-parameter bifurcations in planar Filippov systems," Internat. J. Bifur. Chaos Appl. Sci. Engrg. 13, 2157-2188, doi:10.1142/S0218127403007874, URL http://dx.doi.org/10.1142/S0218127403007874.

Llibre, J. [2008] "Open problems on the algebraic limit cycles of planar polynomial vector fields," Bul. Acad. Ştiinţe Repub. Mold. Mat. , 19-26.

Llibre, J. \& Ponce, E. [2012] "Three nested limit cycles in discontinuous piecewise linear differential systems with two zones," Dyn. Contin. Discrete Impuls. Syst. Ser. B Appl. Algorithms 19, 325-335.

Llibre, J. \& Świrszcz, G. [2006] "Relationships between limit cycles and algebraic invariant curves for quadratic systems," J. Differential Equations 229, 529-537, doi:10.1016/j.jde.2006.03.013, URL http: //dx.doi.org/10.1016/j.jde.2006.03.013.

Llibre, J. \& Świrszcz, G. [2007] "Classification of quadratic systems admitting the existence of an algebraic limit cycle," Bull. Sci. Math. 131, 405-421, doi:10.1016/j.bulsci.2006.03.014, URL http://dx.doi.org/ 10.1016/j.bulsci.2006.03.014.

Llibre, J., Teixeira, M. A. \& Torregrosa, J. [2013] "Lower bounds for the maximum number of limit cycles of discontinuous piecewise linear differential systems with a straight line of separation," Internat. J. Bifur. Chaos Appl. Sci. Engrg. 23, 1350066, 10, doi:10.1142/S0218127413500661, URL http://dx.doi. org/10.1142/S0218127413500661.

Llibre, J. \& Zhang, X. [2016] "Limit cycles for discontinuous planar piecewise linear differential systems," Preprint.

Novaes, D. D., Teixeira, M. A. \& Zeli, I. O. [2018] "Dynamics of Filippov systems near a typical cycle," To appear in Nonlinearity. 